\title{
Kick-Fukui: A Fukui Function-guided Method for Molecular Structure Prediction
}

Osvaldo Yañez, ${ }^{1,2,3}$ Rodrigo Báez-Grez, ${ }^{3}$ Diego Inostroza, ${ }^{3,4}$ Ricardo Pino-Rios, ${ }^{5}$ Walter A. Rabanal-León, ${ }^{6}$ Julia Contreras-García, ${ }^{7 *}$ Carlos Cardenas $^{8,9^{*}}$ and William Tiznado ${ }^{3 *}$

${ }^{1}$ Center of New Drugs for Hypertension (CENDHY), 8380494 Santiago, Chile.

${ }^{2}$ Department of Pharmaceutical Science and Technology, School of Chemical and Pharmaceutical Sciences, Universidad de Chile, 8380494 Santiago, Chile.

${ }^{3}$ Computational and Theoretical Chemistry Group, Departamento de Ciencias Químicas, Facultad de Ciencias Exactas, Universidad Andrés Bello, República 498, 8370035 Santiago, Chile.

${ }^{4}$ Universidad Andres Bello, Programa de Doctorado en Fisicoquímica Molecular, Facultad de Ciencias Exactas, 8370035 Santiago, Chile.

${ }^{5}$ Laboratorio de Química Teórica, Facultad de Química y Biología, Universidad de Santiago de Chile (USACH), 8320000 Santiago, Chile

${ }^{6}$ Departamento de Química Analítica e Inorgánica, Facultad de Ciencias Químicas, Universidad de Concepción, Edmundo Larenas 129, Casilla 160-C, 4070371 Concepción, Chile.

${ }^{7}$ Sorbonne Université and CNRS, Laboratoire de Chimie Théorique (LCT), 75005 Paris, France.

${ }^{8}$ Departamento de Física, Facultad de Ciencias, Universidad de Chile, Casilla, 635, 7790681 Santiago, Chile.

${ }^{9}$ Centro para el Desarrollo de la Nanociencias y Nanotecnologia, CEDENNA, Avenida Ecuador 3493, 9170124 Santiago, Chile. 
The following approximation for the total interaction energy of a donor and acceptor has been deduced elsewhere: ${ }^{1-3}$

$$
\begin{aligned}
& \Delta W_{\text {int }}=\left(\mu_{\text {acceptor }}-\mu_{\text {donor }}\right) \Delta \mathrm{N} \\
&+\int\left(\sum_{\alpha \in \text { donor }} Z_{\alpha} \delta\left(\vec{r}-R_{\alpha}\right)\right. \\
&\left.-\rho_{\text {donor }}(\vec{r})\right) \Phi_{\text {acceptor }}(\vec{r}) d \vec{r} \\
&+\Delta \mathrm{N} \int\left(f_{\text {acceptor }}^{+}\left(\vec{r}^{\prime}\right) \Phi_{\text {donor }}(\vec{r})\right. \\
&\left.-f_{\text {donor }}^{-}(\vec{r}) \Phi_{\text {acceptor }}(\vec{r})\right) d \vec{r} \\
&-(\Delta N)^{2} \iint \frac{f_{\text {donor }}^{-}(\vec{r}) f_{\text {acceptor }}^{+}(\vec{r})}{\left|\vec{r}-\overrightarrow{r^{\prime}}\right|} d \vec{r} d \vec{r}^{\prime}
\end{aligned}
$$

In Eq. S1, donor and acceptor stand for donor and acceptor reagents properties, and $\alpha$ identifies the charge and label of the nuclei. The chemical potential, $\mu$, determines the acceptor/donor reagent, the one with the most/less negative $\mu . \Delta \mathrm{N}$ is the number of electrons transferred from the donor to the acceptor. $\delta\left(\vec{r}-R_{\alpha}\right)$ is the Dirac $\delta$ function centered at the position of the nucleus $\alpha$, which accounts for the electrostatic interaction between the nuclei of both species, which are considered point charges. $\Phi(\mathrm{r})$ is the molecular electrostatic potential. The first term in Eq. 4 is independent of the molecule's orientation and does not play a role in regioselectivity. The second term is a pure electrostatic interaction, and it is generally either small for reagents with noncharged sites (atoms) or negative for reagents with charged sites (atoms). Thus, this term must be tiny in neutral homonuclear clusters because the atomic charge is expected to be negligible. The third term is the correction to the electrostatic interaction because of the electron transfer between both molecules. This term is usually positive: electron transfer weakens the strength of the electrostatic interaction because electron flow from the donor to the acceptor tends to equalize the charges of the reactive sites. Finally, the integrand in the last term is usually positive because the Fukui function is positive, with some exceptions only in small regions around the nuclei of molecules with nodes of the frontier orbitals in the nuclear positions. ${ }^{4,5}$ This term, then, stabilizes and depends entirely on the relative orientation between the "frontier" densities of the molecules; ${ }^{1}$ this term is decisive for explaining the regioselectivity of interacting molecules. Therefore, the best orientation that optimizes the reactants' interaction is enclosed in this term: the orientation must be such that the integrand is a maximum. 
Table S1 - Chemical potential and Cartesian coordinates of the building's blocks used in $\mathrm{Si}_{\mathrm{n}}$ PES exploration (optimized structures at PBE/Def2-TZVP level).

\begin{tabular}{|c|c|c|c|c|c|c|}
\hline $\mathbf{N}^{\circ}$ & $\mu$ & Image & \multicolumn{4}{|c|}{ Cartesian coordinates } \\
\hline $\mathbf{S i}_{2}$ & -0.1940 & & $\begin{array}{l}14 \\
14\end{array}$ & $\begin{array}{r}1.142844398 \\
-1.142844398\end{array}$ & $\begin{array}{l}0.000000000 \\
0.000000000\end{array}$ & $\begin{array}{l}0.000000000 \\
0.000000000\end{array}$ \\
\hline $\mathbf{S i}_{3}$ & -0.1902 & & $\begin{array}{l}14 \\
14 \\
14\end{array}$ & $\begin{array}{l}0.000000000 \\
0.000000000 \\
0.000000000\end{array}$ & $\begin{array}{r}1.571695710 \\
0.000000000 \\
-1.571695710\end{array}$ & $\begin{array}{r}-0.533522895 \\
1.067045255 \\
-0.533522895\end{array}$ \\
\hline $\mathbf{S i}_{4}$ & -0.1934 & & $\begin{array}{l}14 \\
14 \\
14 \\
14\end{array}$ & $\begin{array}{l}0.000000000 \\
0.000000000 \\
0.000000000 \\
0.000000000\end{array}$ & $\begin{array}{r}1.276157070 \\
0.000000000 \\
0.000000000 \\
-1.276157070\end{array}$ & $\begin{array}{r}0.000000000 \\
2.038765574 \\
-2.038765574 \\
0.000000000\end{array}$ \\
\hline $\mathbf{S i}_{5}$ & -0.1865 & & $\begin{array}{l}14 \\
14 \\
14 \\
14 \\
14\end{array}$ & $\begin{array}{r}0.000000000 \\
-1.651465127 \\
0.000000000 \\
1.651465127 \\
0.000000000\end{array}$ & $\begin{array}{r}1.906948146 \\
-0.953474070 \\
0.000000000 \\
-0.953474070 \\
0.000000000\end{array}$ & $\begin{array}{r}0.000000000 \\
0.000000000 \\
1.505143114 \\
0.000000000 \\
-1.505143114\end{array}$ \\
\hline $\mathbf{S i}_{6}$ & -0.1831 & & $\begin{array}{l}14 \\
14 \\
14 \\
14 \\
14 \\
14\end{array}$ & $\begin{array}{r}0.385691925 \\
-0.240855043 \\
-2.219366377 \\
-0.241023321 \\
1.922950894 \\
0.392601923\end{array}$ & $\begin{array}{r}2.035592627 \\
-0.000659884 \\
-0.003076107 \\
-0.000705922 \\
0.003375622 \\
-2.034526864\end{array}$ & $\begin{array}{r}-0.000241834 \\
-1.415652966 \\
0.000157166 \\
1.415606927 \\
0.000404291 \\
-0.000273585\end{array}$ \\
\hline $\mathbf{S i}_{7}$ & -0.1952 & & $\begin{array}{l}14 \\
14 \\
14 \\
14 \\
14 \\
14 \\
14\end{array}$ & $\begin{array}{r}0.000000000 \\
0.000000000 \\
-1.380460033 \\
0.000000000 \\
0.000000000 \\
0.000000000 \\
1.380460033\end{array}$ & $\begin{array}{r}0.000000000 \\
2.115090407 \\
0.000000000 \\
-2.115090407 \\
-1.307200200 \\
1.307200200 \\
0.000000000\end{array}$ & $\begin{array}{r}2.223940585 \\
0.687229846 \\
0.000000000 \\
0.687229846 \\
-1.799200138 \\
-1.799200138 \\
0.000000000\end{array}$ \\
\hline
\end{tabular}




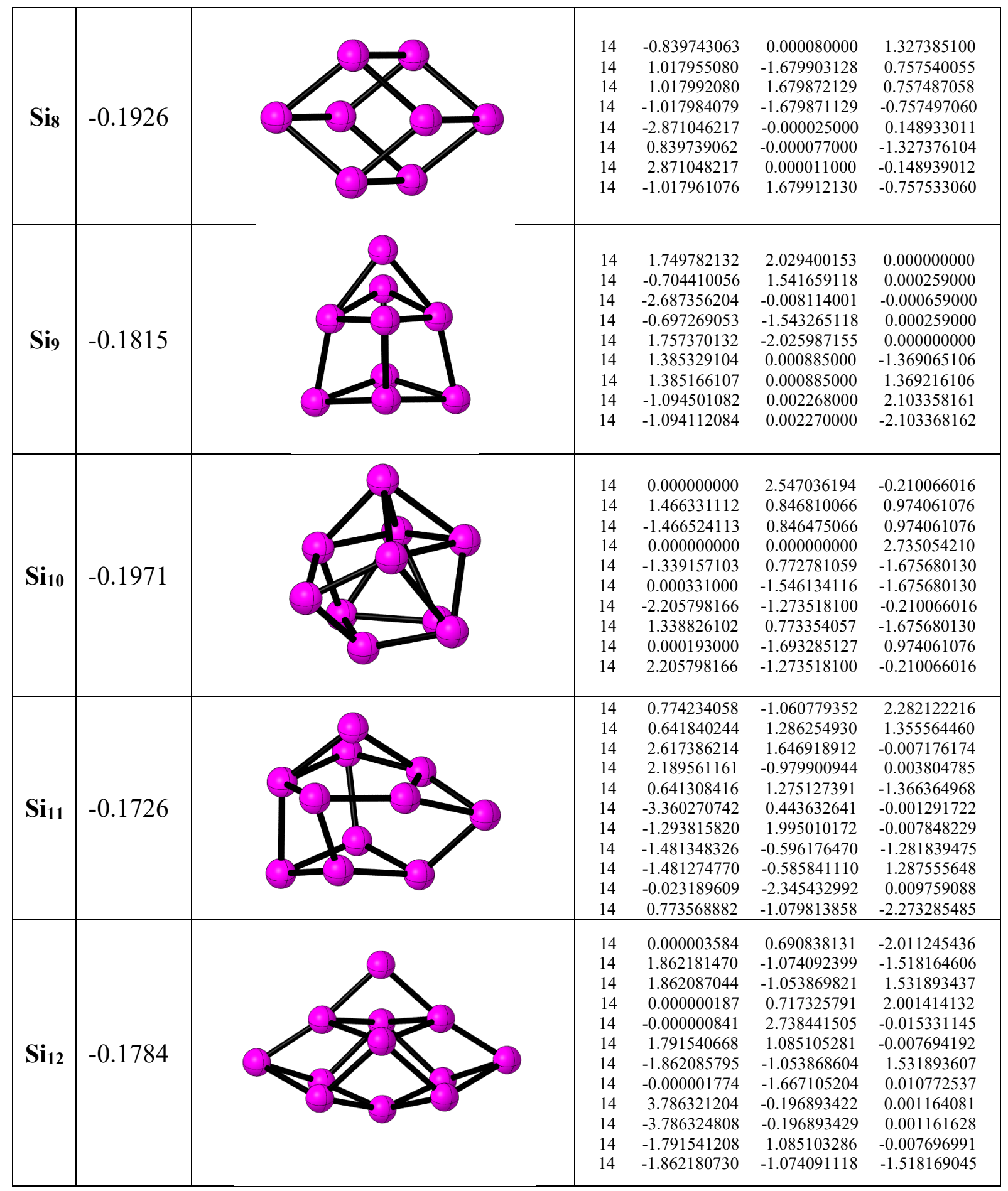




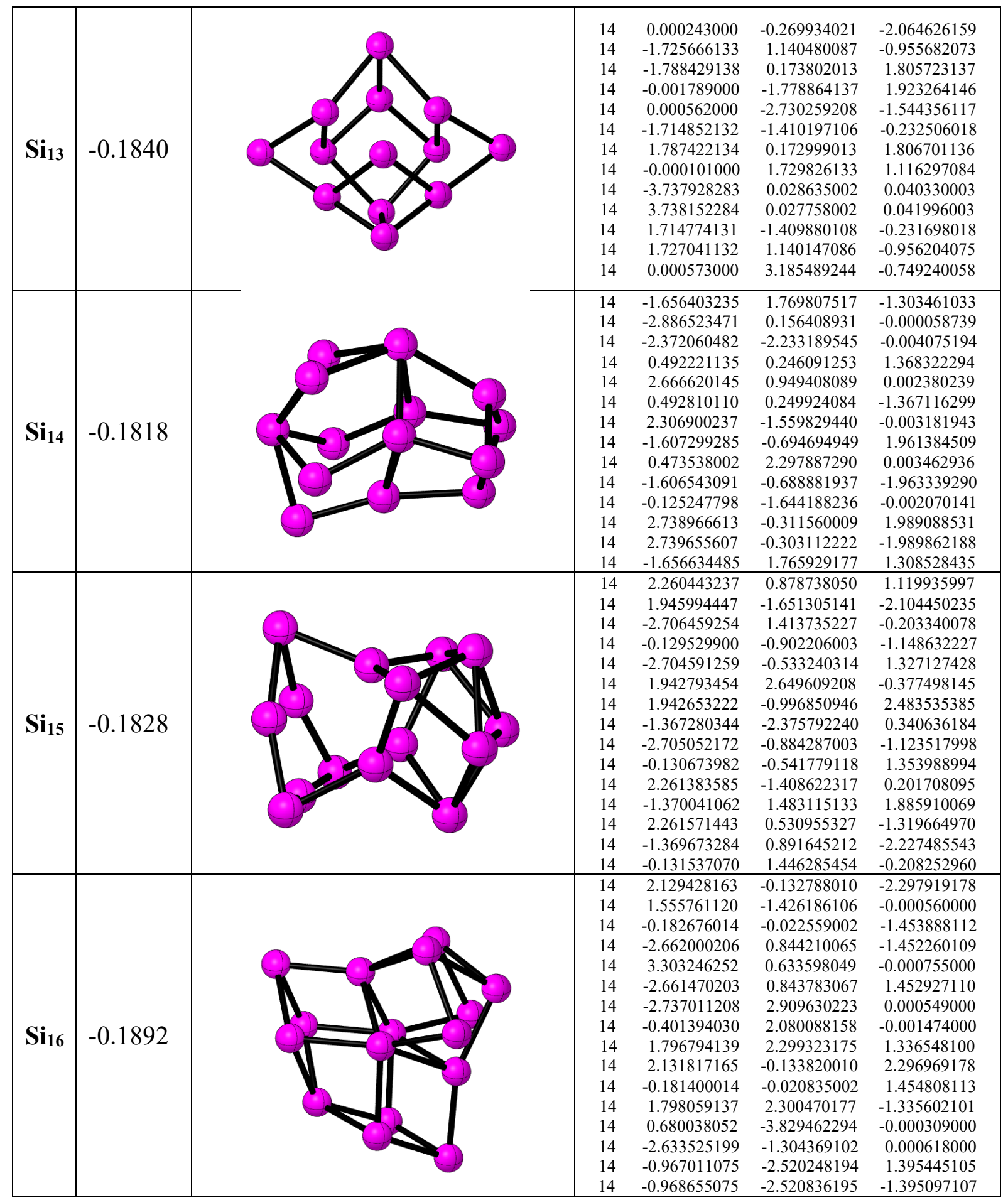




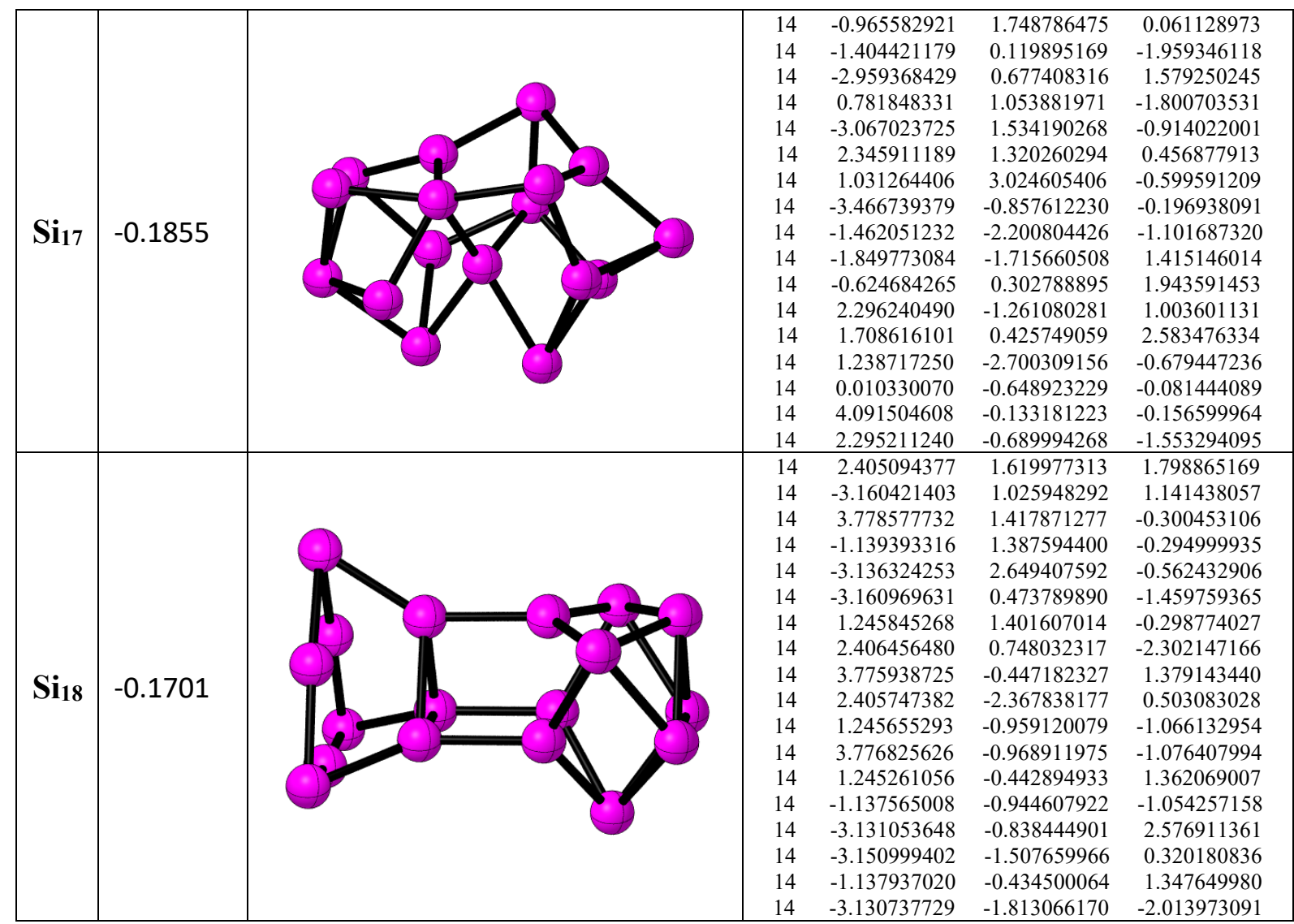


Table S2 - All possible combinations used in the $\mathrm{Si}_{\mathrm{n}}(\mathrm{n}=12-20)$ PES exploration. In bold are identified the assemblies that converge to the best energy structure.

\begin{tabular}{|c|c|c|}
\hline & $f^{+}$ & $f^{-}$ \\
\hline \multirow{5}{*}{$\mathbf{S i}_{12}$} & $\mathrm{Si}_{3}$ & Sig \\
\hline & $\mathrm{Si}_{4}$ & $\mathrm{Si}_{8}$ \\
\hline & $\mathrm{Si}_{6}$ & $\mathrm{Si}_{6}$ \\
\hline & $\mathrm{Si}_{7}$ & $\mathrm{Si}_{5}$ \\
\hline & $\mathrm{Si}_{10}$ & $\mathrm{Si}_{2}$ \\
\hline \multirow{5}{*}{$\mathbf{S i}_{13}$} & $\mathrm{Si}_{2}$ & $\mathrm{Si}_{11}$ \\
\hline & $\mathbf{S i}_{4}$ & Sig \\
\hline & $\mathrm{Si}_{7}$ & $\mathrm{Si}_{6}$ \\
\hline & $\mathrm{Si}_{8}$ & $\mathrm{Si}_{5}$ \\
\hline & $\mathrm{Si}_{10}$ & $\mathrm{Si}_{3}$ \\
\hline \multirow{6}{*}{$\mathbf{S i}_{14}$} & $\mathrm{Si}_{2}$ & $\mathrm{Si}_{12}$ \\
\hline & $\mathrm{Si}_{3}$ & $\mathrm{Si}_{11}$ \\
\hline & $\mathrm{Si}_{5}$ & $\mathrm{Si}_{9}$ \\
\hline & $\mathrm{Si}_{7}$ & $\mathrm{Si}_{7}$ \\
\hline & $\mathrm{Si}_{8}$ & $\mathrm{Si}_{6}$ \\
\hline & $\mathbf{S i}_{10}$ & $\mathbf{S i}_{4}$ \\
\hline \multirow{6}{*}{$\mathbf{S i}_{15}$} & $\mathbf{S i}_{2}$ & $\mathbf{S i}_{13}$ \\
\hline & $\mathrm{Si}_{3}$ & $\mathrm{Si}_{12}$ \\
\hline & $\mathrm{Si}_{4}$ & $\mathrm{Si}_{11}$ \\
\hline & $\mathrm{Si}_{6}$ & $\mathrm{Si}_{9}$ \\
\hline & $\mathrm{Si}_{7}$ & $\mathrm{Si}_{8}$ \\
\hline & $\mathrm{Si}_{10}$ & $\mathrm{Si}_{5}$ \\
\hline \multirow{7}{*}{$\mathbf{S i}_{16}$} & $\mathrm{Si}_{2}$ & $\mathrm{Si}_{14}$ \\
\hline & $\mathrm{Si}_{3}$ & $\mathrm{Si}_{13}$ \\
\hline & $\mathrm{Si}_{4}$ & $\mathrm{Si}_{12}$ \\
\hline & $\mathrm{Si}_{5}$ & $\mathrm{Si}_{11}$ \\
\hline & $\mathrm{Si}_{6}$ & $\mathrm{Si}_{10}$ \\
\hline & $\mathrm{Si}_{8}$ & $\mathrm{Si}_{8}$ \\
\hline & $\mathbf{S i}_{10}$ & $\mathbf{S i}_{6}$ \\
\hline \multirow{7}{*}{$\mathbf{S i}_{17}$} & $\mathrm{Si}_{2}$ & $\mathrm{Si}_{15}$ \\
\hline & $\mathrm{Si}_{3}$ & $\mathrm{Si}_{14}$ \\
\hline & $\mathrm{Si}_{4}$ & $\mathbf{S i}_{13}$ \\
\hline & $\mathrm{Si}_{5}$ & $\mathrm{Si}_{12}$ \\
\hline & $\mathrm{Si}_{6}$ & $\mathrm{Si}_{11}$ \\
\hline & $\mathrm{Si}_{8}$ & $\mathrm{Si}_{9}$ \\
\hline & $\mathrm{Si}_{10}$ & $\mathrm{Si}_{7}$ \\
\hline \multirow{6}{*}{$\mathbf{S i}_{18}$} & $\mathrm{Si}_{2}$ & $\mathrm{Si}_{16}$ \\
\hline & $\mathrm{Si}_{3}$ & $\mathrm{Si}_{15}$ \\
\hline & $\mathrm{Si}_{4}$ & $\mathrm{Si}_{14}$ \\
\hline & $\mathrm{Si}_{5}$ & $\mathrm{Si}_{13}$ \\
\hline & $\mathrm{Si}_{6}$ & $\mathrm{Si}_{12}$ \\
\hline & $\mathrm{Si}_{7}$ & $\mathrm{Si}_{11}$ \\
\hline
\end{tabular}




\begin{tabular}{|c|c|c|}
\hline & $\begin{array}{c}\mathbf{S i}_{9} \\
\mathrm{Si}_{10}\end{array}$ & $\begin{array}{l}\mathbf{S i}_{9} \\
\mathrm{Si}_{8}\end{array}$ \\
\hline \multirow{8}{*}{$\mathbf{S i}_{19}$} & $\mathrm{Si}_{2}$ & $\mathrm{Si}_{17}$ \\
\hline & $\mathrm{Si}_{3}$ & $\mathrm{Si}_{16}$ \\
\hline & $\mathrm{Si}_{4}$ & $\mathrm{Si}_{15}$ \\
\hline & $\mathrm{Si}_{5}$ & $\mathrm{Si}_{14}$ \\
\hline & $\mathrm{Si}_{7}$ & $\mathrm{Si}_{12}$ \\
\hline & $\mathrm{Si}_{8}$ & $\mathrm{Si}_{11}$ \\
\hline & $\mathbf{S i}_{10}$ & Sig \\
\hline & $\mathrm{Si}_{13}$ & $\mathrm{Si}_{6}$ \\
\hline \multirow{9}{*}{$\mathbf{S} \mathbf{i}_{20}$} & $\mathrm{Si}_{2}$ & $\mathrm{Si}_{18}$ \\
\hline & $\mathrm{Si}_{3}$ & $\mathrm{Si}_{17}$ \\
\hline & $\mathrm{Si}_{4}$ & $\mathrm{Si}_{16}$ \\
\hline & $\mathrm{Si}_{5}$ & $\mathrm{Si}_{15}$ \\
\hline & $\mathrm{Si}_{6}$ & $\mathrm{Si}_{14}$ \\
\hline & $\mathrm{Si}_{7}$ & $\mathrm{Si}_{13}$ \\
\hline & $\mathrm{Si}_{8}$ & $\mathrm{Si}_{12}$ \\
\hline & $\mathrm{Si}_{9}$ & $\mathrm{Si}_{11}$ \\
\hline & $\mathbf{S i}_{10}$ & $\mathbf{S i}_{10}$ \\
\hline
\end{tabular}


Table S3 - Structures and Cartesian coordinates of minimum energy structures for $\mathrm{Si}_{\mathrm{n}}$ $(n=12-20)$ at PBE0/Def2-TZVP identified by Kick-Fukui method.

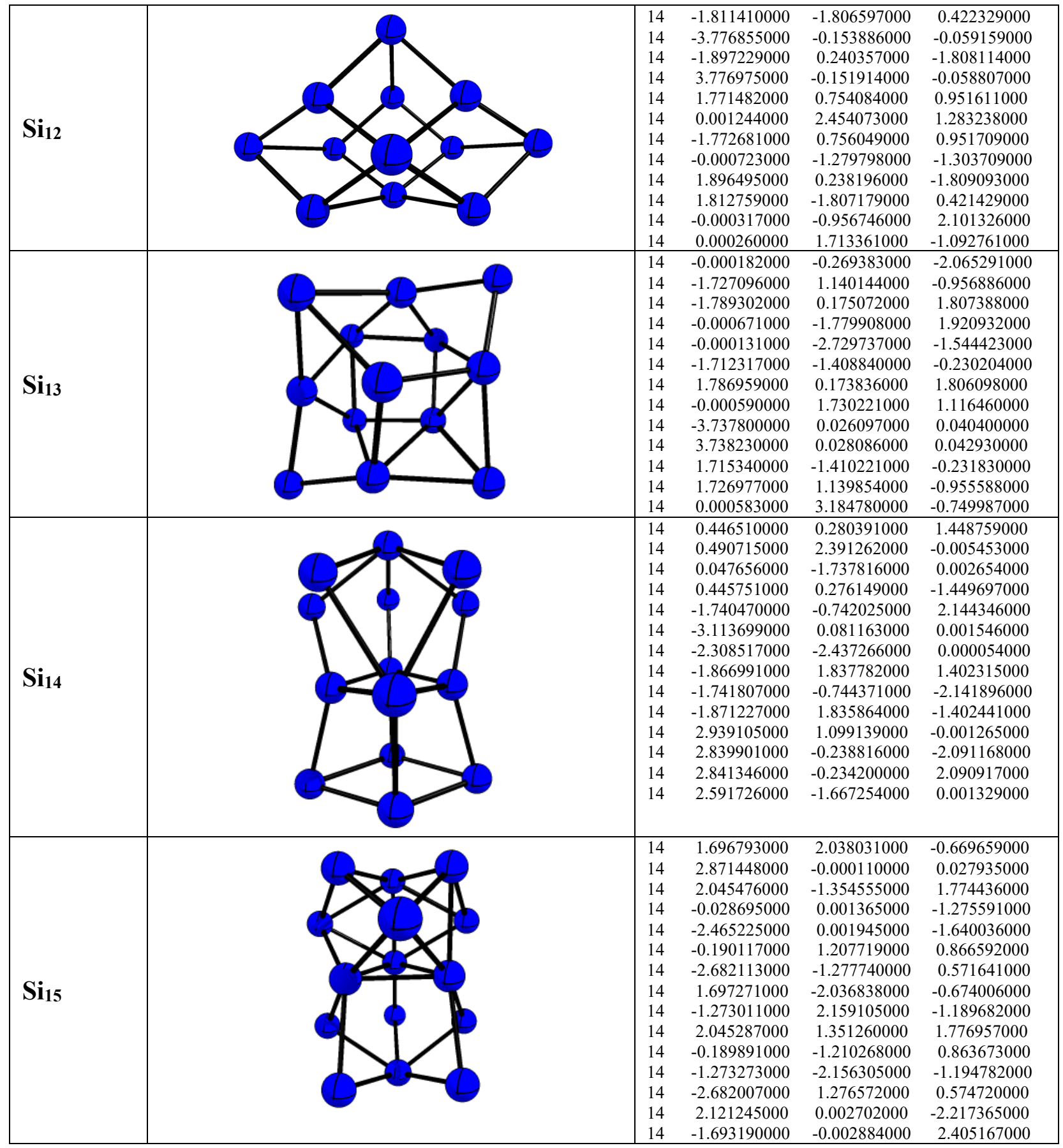




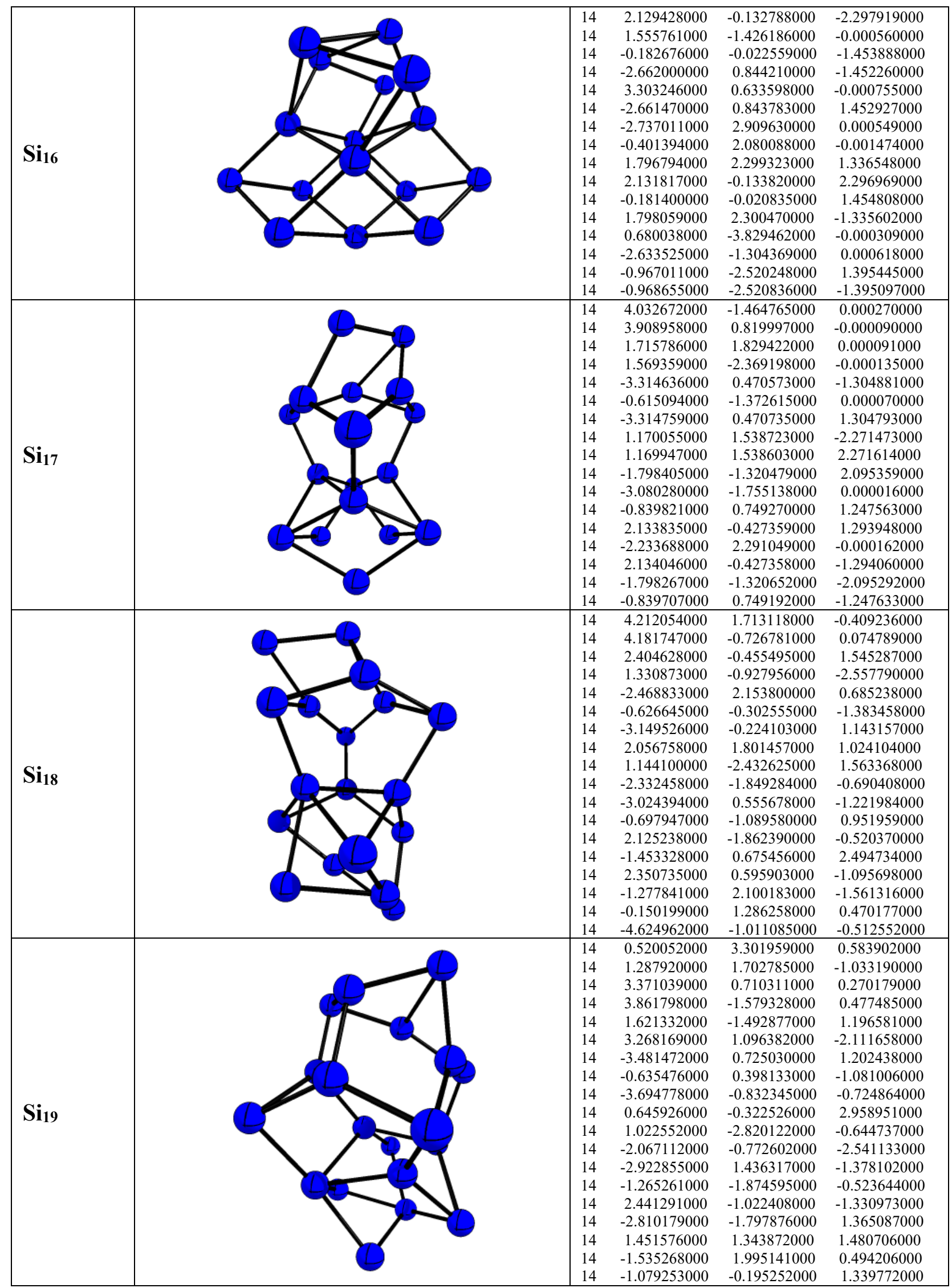




Sín




\section{References}

(1) Berkowitz, M. Density Functional Approach to Frontier Controlled Reactions. J. Am. Chem. Soc. 1987, 109 (16), 4823-4825.

(2) Ayers, P. W.; Parr, R. G.; Pearson, R. G. Elucidating the Hard/Soft Acid/Base Principle: A Perspective Based on Half-Reactions. J. Chem. Phys. 2006, 124 (19), 194107.

(3) Ayers, P. W. The Physical Basis of the Hard/Soft Acid/Base Principle. Faraday Discuss. 2007, 135, 161-190.

(4) Melin, J.; Ayers, P. W.; Ortiz, J. V. Removing Electrons Can Increase the Electron Density: A Computational Study of Negative Fukui Functions. J. Phys. Chem. A 2007, 111 (40), 10017-10019.

(5) Bultinck, P.; Clarisse, D.; Ayers, P. W.; Carbo-Dorca, R. The Fukui Matrix: A Simple Approach to the Analysis of the Fukui Function and Its Positive Character. Phys. Chem. Chem. Phys. 2011, 13 (13), 6110-6115. 